\title{
Complexity of Cycle Length Modularity Problems in Graphs*
}

\author{
Edith Hemaspaandra \\ Department of Computer Science \\ Rochester Institute of Technology \\ Rochester, NY 14623, USA \\ eh@cs.rit.edu.
}

\author{
Holger Spakowski ${ }^{\dagger}$ \\ Institut für Informatik \\ Heinrich-Heine-Universität Düsseldorf \\ 40225 Düsseldorf, Germany \\ spakowsk@cs.uni-duesseldorf.de.
}

\author{
Mayur Thakur \\ Department of Computer Science \\ University of Rochester \\ Rochester, NY 14627, USA \\ thakur@cs.rochester.edu.
}

June 25, 2003

\begin{abstract}
The even cycle problem for both undirected Tho88 and directed [RST99 graphs has been the topic of intense research in the last decade. In this paper, we study the computational complexity of cycle length modularity problems. Roughly speaking, in a cycle length modularity problem, given an input (undirected or directed) graph, one has to determine whether the graph has a cycle $C$ of a specific length (or one of several different lengths), modulo a fixed integer. We denote the two families (one for undirected graphs and one for directed graphs) of problems by $(S, m)$-UC and $(S, m)$-DC, where $m \in \mathbb{N}$ and $S \subseteq\{0,1, \ldots, m-1\}$. $(S, m)$-UC (respectively, $(S, m)$-DC) is defined as follows: Given an undirected (respectively, directed) graph $G$, is there a cycle in $G$ whose length, modulo $m$, is a member of $S$ ? In this paper, we fully classify (i.e., as either polynomial-time solvable or as NP-complete) each problem $(S, m)$-UC such that $0 \in S$ and each problem $(S, m)$-DC such that $0 \notin S$. We also give a sufficient condition on $S$ and $m$ for the following problem to be polynomial-time computable: $(S, m)$-UC such that $0 \notin S$.
\end{abstract}

\section{Introduction}

In this paper we study the complexity of problems related to lengths of cycles, modulo a fixed integer, in undirected and directed graphs. Given $m \in \mathbb{N}$, and $S \subseteq\{0,1, \ldots, m-1\}$, we define the following two cycle length modularity problems.

$(S, m)-\mathrm{UC}=\{G \mid G$ is an undirected graph such that there exists an $\ell \in \mathbb{N}$ such that $\ell \bmod m \in S$, and there exists a cycle of length $\ell$ in $G\}$.

$(S, m)$-DC $=\{G \mid G$ is an directed graph such that there exists an $\ell \in \mathbb{N}$ such that $\ell \bmod m \in S$, and there exists a directed cycle of length $\ell$ in $G\}$.

\footnotetext{
* Supported in part by grants NSF-INT-9815095/DAAD-315-PPP-gü-ab, NSF-CCR-0311021, and a DAAD grant.

${ }^{\dagger}$ Work done while visiting the University of Rochester.
} 
The most basic cases of cycle length modularity problems are the following problems for undirected (respectively, directed) graphs: deciding whether a given undirected (respectively, directed) graph has a cycle of odd length, and deciding whether a given undirected (respectively, directed) graph has a cycle of even length. We will refer to these problems as the odd cycle problem for undirected (respectively, directed) graphs, and the even cycle problem for undirected (respectively, directed) graphs, respectively. In our notation, these problems are denoted by $(\{1\}, 2)-\mathrm{UC}$ (respectively, $(\{1\}, 2)-\mathrm{DC})$ and $(\{0\}, 2)-\mathrm{UC}$ (respectively, $(\{0\}, 2)-\mathrm{DC})$. All these four problems are now known to be in $\mathrm{P}$.

The odd cycle problem and the even cycle problem are quite different in nature. The reason is that if a closed walk of odd length is decomposed into cycles, then there is at least one odd cycle in the decomposition. The corresponding statement for even walks and even cycles is not true. Since odd closed walks can easily be found in polynomial time, it is easy to detect odd cycles.

It is well known that an undirected graph has an odd cycle if and only if the graph is not bipartite. No such simple characterization is known for the case of even cycles. However, Thomassen Tho88 showed that the family of cycles of length divisible by $m$ has the Erdös-Pósa property [EP65], and then used results from Robertson and Seymour RS86 to prove that the even cycle problem for undirected graphs is in P. In fact, Thomassen proved that, for each $m \in \mathbb{N},(\{0\}, m)$-UC is in P. Even though Thomassen's graph minor and tree-width approach to solving the even cycle problem is elegant, it means that the algorithm has the drawback of having huge constants in its running time. Arkin, Papadimitriou, and Yannakakis APY91 used a simpler approach to give an efficient algorithm for the even cycle problem for undirected graphs. Their algorithm is based on their characterization of undirected graphs that do not contain even cycles with certain efficiently checkable properties of the biconnected components of these graphs. However, unlike Thomassen's approach, their approach does not seem to generalize beyond the $(\{0\}, 2)$-UC case to, say $(\{0\}, 3)-\mathrm{UC}$. A related result from Yuster and Zwick [YZ97] shows that, for each $k$, the problem of deciding if a given undirectd graph has a cycle of length $2 k$ is in $\mathrm{P}$.

It is interesting to note that even though the algorithms for the two odd cycle problems (i.e., undirected and directed) are similar, neither of the two algorithms mentioned above (namely, Thomassen Tho88 and Arkin, Papadimitriou, and Yannakakis APY91) seems to be able to handle the even cycles case for directed graphs. However, Robertson, Seymour, and Thomas [RST99] (the conference version of the paper is by McCuaig et al. [MRST97]) prove, via giving a polynomial time algorithm for the problem of deciding whether a bipartite graph has a Pfaffian orientation, that the even cycle problem for directed graphs is also in P. We note that Vazirani and Yannakakis VY89 proved the polynomial-time equivalence of the even cycle problem for directed graphs with the following problems:

1. The problem of checking whether a bipartite graph has a Pfaffian orientation Kas67,

2. Polya's problem Pol13, i.e., given a square $(0,1)$ matrix $A$, is there a $(-1,0,1)$ matrix $B$ that can be obtained from $A$ by changing some of the 1's to -1 's such that the determinant of $B$ is equal to the permanent of $A$, and

3. Given a square matrix $A$ of nonnegative integers, determine if the determinant of $A$ equals the permanent of $A$.

Until the even cycle problem was shown to be in P by Thomassen Tho88 (for undirected graphs) and Robertson, Seymour, and Thomas RST99 (for directed graphs) and even since then, a lot of interesting research on the even cycle problem and related problems led to the study of other cycle length modularity problems. Some problems have been shown to be in $\mathrm{P}$, while others have been shown to be NP-complete. We mention some that have a close relationship with the problems studied in this paper. As stated earlier, Thomassen Tho88 proved that, for each $m \in \mathbb{N}$, the problem of 
deciding whether an undirected graph has a cycle of length $\equiv 0(\bmod m)$ is in $\mathrm{P}$. That is, for each $m \in \mathbb{N},(\{0\}, m)-\mathrm{UC} \in \mathrm{P}$. Arkin, Papadimitriou, and Yannakakis APY91 study, among other problems, the problem of deciding whether a directed graph contains cycles of length $p(\bmod m)$. They prove, via reduction from the directed subgraph homeomorphism problem FHW80, that for all $m>2$ and for all $p$ such that $0<p<m,(\{p\}, m)$-DC is NP-complete. They also give a polynomial-time algorithm for the problem of finding the greatest common divisor of all cycles in graphs, a problem motivated by the problem of finding the period of a Markov chain. Furthermore, they prove that, for all $m>2$ and for all $0<p<m$, the problem of deciding whether all cycles in an undirected graph are of length $p(\bmod m)$ is in P. Galluccio and Loebl GL96] study the complexity of the corresponding problem in directed graphs. They prove that for the case of planar directed graphs, checking whether all cycles in the input graph are of length $p(\bmod m)$ can be done in polynomial time.

In this paper, we resolve the complexity (as either in P or NP-complete) of the following problems:

1. $(S, m)$-DC, where $0 \notin S$, and

2. $(S, m)$-UC, where $0 \in S$.

We prove that each problem in 2 is in $\mathrm{P}$. For 1 we classify each problem as either in $\mathrm{P}$ or NP-complete, depending only on the properties of $S$ and $m$ : If there exist $0 \leq d_{1}, d_{2}<m$ such that $d_{1}, d_{2} \notin S$ and $\left(d_{1}+d_{2}\right)$ mod $m \in S$, then $(S, m)$-DC is NP-complete, otherwise $(S, m)$-DC is in P. We also prove a sufficient condition for $(S, m)$-UC (with $0 \notin S$ ) to be polynomial-time computable: for each $p \in S$, and for each $d_{1}, d_{2}$ such that $0 \leq d_{1}, d_{2}<m$ and $d_{1}+d_{2} \equiv p(\bmod m)$, it holds that either $d_{1} \in S$ or $d_{2} \in S$. Note that this condition is exactly the same as that for the "in P" case of 1 given above.

The paper is organized as follows. In Section 2 we introduce the definitions and notations that will be used in the rest of the paper. In Section [3 we present results for cycle length modularity problems in directed graphs and in Section 4 we present results for cycle length modularity problems in undirected graphs. Finally, in Section [5 we present some open problems and future research directions.

\section{Definitions and Notations}

In this section we describe the notations used in the rest of the paper. For each finite set $S$, let $\|S\|$ denote the cardinality of $S$.

Since this paper is about graphs, let us make the notations very clear. An undirected graph $G$ is a pair $(V, E)$, where $V$ is a finite set (known as the set of vertices or nodes) and $E \subseteq V \times V$ (known as the set of edges) with the following properties.

1. For each $u, v \in V$, if $(u, v) \in E$, then $(v, u) \in E$.

2. For each $v \in V,(v, v) \notin E$, that is, self-loops are not allowed.

A directed graph $G$ is a pair $(V, E)$, where $V$ is a finite set and $E \subseteq V \times V$. For each graph $G$, let $V(G)$ denote the set of vertices of $G$, and let $E(G)$ denote the set of edges of $G$. A walk of length $k$ in a graph $G$ is a sequence of vertices $\left(u_{0}, u_{1}, \ldots, u_{k}\right)$ with $k \geq 1$ in $G$ such that, for each $0 \leq i<k,\left(u_{i}, u_{i+1}\right) \in E(G)$. A path is a walk where all vertices are distinct. A closed walk in a graph $G$ is a walk $\left(u_{0}, u_{1}, \ldots, u_{k}\right)$ in $G$ such that $u_{0}=u_{k}$. A cycle in an undirected graph is a closed walk $\left(u_{0}, u_{1}, \ldots, u_{k-1}, u_{0}\right)$ of length $\geq 3$ such that $u_{0}, u_{1}, \ldots, u_{k-1}$ are $k$ distinct vertices. A cycle in a directed graph is a closed walk $\left(u_{0}, u_{1}, \ldots, u_{k-1}, u_{0}\right)$ such that $u_{0}, u_{1}, \ldots, u_{k-1}$ are $k$ distinct vertices. It should be noted that this definition of a cycle is sometimes called a simple cycle. 


\section{Cycle Length Modularity Problems in Directed Graphs}

In this section, we study the complexity of cycle length modularity problems in directed graphs. Arkin, Papadimitriou, and Yannakakis APY91 proved that, for each $m \in \mathbb{N}$ and each $0<r<m$, $(\{r\}, m)$-DC is NP-complete. In Theorem 3.1 we generalize their result. For each $m$ and $S$ such that $0 \notin S$, we give a condition on $S$ and $m$ for $(S, m)$-DC to be NP-complete. Furthermore, we prove that if the stated conditions on $S$ and $m$ are not satisfied, then $(S, m)-\mathrm{DC}$ is in $\mathrm{P}$.

Theorem 3.1 For all $m \geq 1$ and $S \subseteq\{1, \ldots, m-1\}$, the following is true:

(i) If there is a $p \in S$, and $d_{1} \notin S, d_{2} \notin S$ such that $0 \leq d_{1}, d_{2}<m$ and $d_{1}+d_{2} \equiv p(\bmod m)$, then $(S, m)$-DC is NP-complete.

(ii) Otherwise, $(S, m)-\mathrm{DC}$ is in $\mathrm{P}$.

Proof To prove (i), let $p \in S$ and $d_{1}, d_{2} \notin S$ be such that $0 \leq d_{1}, d_{2}<m$ and $d_{1}+d_{2} \equiv p(\bmod m)$. We closely follow the proof of Theorem 1 in Arkin, Papadimitriou, and Yannakakis APY91. Fortune, Hopcroft, and Wyllie [FHW80] showed that the directed subgraph homeomorphism problem is NP-complete for any fixed directed graph that is not a tree of depth 1 . In particular, the following problem is NP-complete:

Given a directed graph $G$ and vertices $s$ and $t$ in $V(G)$, does $G$ contain a cycle through both $s$ and $t$ ?

We will prove that $(S, m)$-DC is NP-complete by polynomial-time many-one reducing this problem to $(S, m)$-DC. We now specify a polynomial-time function $\sigma$ that reduces this problem to $(S, m)$-DC. Given a directed graph $G$ and vertices $s, t \in V(G), \sigma(\langle G, s, t\rangle)$ outputs the graph $G^{\prime}$ where $G^{\prime}=$ $\left(V^{\prime}, E^{\prime}\right)$ is defined as follows. $V^{\prime}$ and $E^{\prime}$ are constructed in the following steps. (Note that in the steps below, we can assume that $d_{1} \neq 0$ and $d_{2} \neq 0$, because if either $d_{1}$ or $d_{2}$ is equal to 0 , then the preconditions of (i) cannot be satisfied.)

1. Set $V^{\prime}:=V$. Set $E^{\prime}:=\emptyset$.

2. For every edge $(v, s) \in E(G)$, do the following.

(a) Set $V^{\prime}:=V^{\prime} \cup\left\{w_{j} \mid 1 \leq j \leq d_{1}-1\right\}$, where the $w_{j}$ 's are new vertices.

(b) Set $E^{\prime}:=E^{\prime} \cup\left\{\left(v, w_{1}\right),\left(w_{1}, w_{2}\right), \ldots,\left(w_{d_{1}-1}, s\right)\right\}$.

3. For every edge $(v, t) \in E(G)$, do the following.

(a) Set $V^{\prime}:=V^{\prime} \cup\left\{w_{j} \mid 1 \leq j \leq d_{2}-1\right\}$, where the $w_{j}$ 's are new vertices.

(b) Set $E^{\prime}:=E^{\prime} \cup\left\{\left(v, w_{1}\right),\left(w_{1}, w_{2}\right), \ldots,\left(w_{d_{2}-1}, t\right)\right\}$.

4. For every edge $(v, w) \in E(G)$ such that $v, w \notin\{s, t\}$, do the following.

(a) Set $V^{\prime}:=V^{\prime} \cup\left\{w_{j} \mid 1 \leq j \leq m-1\right\}$, where the $w_{j}$ 's are new vertices.

(b) Set $E^{\prime}:=E^{\prime} \cup\left\{\left(v, w_{1}\right),\left(w_{1}, w_{2}\right), \ldots,\left(w_{m-1}, w\right)\right\}$.

It is easy to see that the cycles in $G^{\prime}$ have the following properties.

1. All cycles in $G^{\prime}$ going through neither $s$ nor $t$ have length $\equiv 0(\bmod m)$.

2. All cycles in $G^{\prime}$ going through $s$ but not through $t$ have length $\equiv d_{1}(\bmod m)$.

3. All cycles in $G^{\prime}$ going through $t$ but not through $s$ have length $\equiv d_{2}(\bmod m)$.

4. All cycles in $G^{\prime}$ going through $s$ and $t$ have length $\equiv\left(d_{1}+d_{2}\right)(\bmod m) \equiv p(\bmod m)$ 
Roughly speaking, we replace each edge $e \in E(G)$ that ends in $s$, by a series of $d_{1}$ edges in $G^{\prime}$ such that the series of edges ends in $s$. Similarly, we replace each edge $e \in E(G)$ that ends in $t$, by a series of $d_{2}$ edges in $G^{\prime}$ that ends in $t$. It is clear from the construction of $G^{\prime}$ that there is a cycle through $s$ and $t$ in $G$ if and only if there is a cycle through $s$ and $t$ in $G^{\prime}$. Since $\left\{0, d_{1}, d_{2}\right\} \cap S=\emptyset$ and $p \in S$, it follows from the properties stated above that there is a cycle through $s$ and $t$ in $G$ if and only if there is a cycle of length $\equiv p(\bmod m)$ in $G^{\prime}$. Also, it is clear that $G^{\prime}$ can be computed from $G$ in polynomial time. It follows that $(S, m)$-DC is NP-hard. Note that, for each $S$ and $m$, $(S, m)$-DC is clearly in NP. Thus, $(S, m)$-DC is NP-complete.

We will now prove (ii). Let $m \geq 1$ and $S \subseteq\{1, \ldots, m-1\}$ be such that for all $p \in S$ and all $d_{1}, d_{2}$, if $0 \leq d_{1}, d_{2}<m$ and $d_{1}+d_{2} \equiv p(\bmod m)$, then $d_{1} \in S$ or $d_{2} \in S$.

We claim that the following algorithm solves $(S, m)$-DC in polynomial time:

Input: A directed graph $G$.

1. for each $p \in S$ do

2. $\quad$ if $G$ has a closed walk of length $\equiv p(\bmod m)$ then accept. 3. reject.

Clearly, step 2 can be done in polynomial time. If the algorithm rejects, then obviously $G$ is not in $(S, m)$-DC. To complete the proof of (ii), we will prove the following claim.

Claim 3.2 If $G$ has a closed walk $W$ of length $\equiv p(\bmod m)$ for some $p \in S$, then $G$ has a cycle of length $\equiv p^{\prime}(\bmod m)$ for some $p^{\prime} \in S$.

Proof The proof is by induction on the length of $W$. The claim is certainly true for all closed walks $W$ of length 1 . Assume that the claim is true for all closed walks $W$ whose length is less than $k$. Suppose $G$ has a closed walk $W$ of length $k$ with $k \bmod m=p$ and $p \in S$. Distinguish the following two cases.

Case 1: $W$ is a cycle.

Then we are done.

Case 2: $W$ is not a cycle.

Then there exist $\ell_{1}>0, \ell_{2}>0, d_{1}<m$, and $d_{2}<m$ such that $W$ can be decomposed into a simple cycle $C$ of length $\ell_{1}$ and a closed walk $W^{\prime}$ of length $\ell_{2}$ such that $\ell_{1} \equiv d_{1}(\bmod m)$, $\ell_{2} \equiv d_{2}(\bmod m)$. Since $\ell_{1}+\ell_{2}=k$, it follows that $\ell_{1}+\ell_{2} \equiv d_{1}+d_{2} \equiv p(\bmod m)$. We know that $d_{1} \in S$ or $d_{2} \in S$. If $d_{1} \in S$ then we are done. If $d_{2} \in S$ we are done by the induction hypothesis.

Thus, Claim 3.2 holds, and so Theorem 3.1 holds.

As an immediate corollary, we get that the problem of deciding whether all cycles in a directed graph have length $\equiv 0(\bmod m)$ is in $\mathrm{P}$.

Corollary 3.3 For each $m \in \mathbb{N},(\{1,2, \ldots, m-1\}, m)$-DC $\in \mathrm{P}$.

We note that Corollary 3.3 also follows from the fact that finding the period (greatest common divisor of all cycle lengths) of a graph is in P BV73 (see also Knu73 Tar72]).

Yuster and Zwick [YZ97] proved that for directed graphs, a shortest odd length cycle can be found in time $O(\|V\| \cdot\|E\|)$. We show that for all $(S, m)$-DC-problems satisfying the condition (ii) of Theorem 3.1 a shortest cycle with length, modulo $m$, in $S$ can be found in time $O(M(\|V\|) \cdot \log \|V\|)$, where $M(n)=n^{2.376}$ is the complexity of boolean matrix multiplication. For the special case $m=2$, $S=\{1\}$, the algorithm is for dense graphs an improvement over the one given in [YZ97.

Theorem 3.4 For all $m \geq 2$ and $S \subseteq\{0, \ldots, m-1\}$ with $0 \notin S$ the following is true: If for all $p \in S$, and all $d_{1}, d_{2}$, such that $0 \leq d_{1}, d_{2}<m$ and $d_{1}+d_{2} \equiv p(\bmod m)$, it holds that $d_{1} \in S$ or 
$d_{2} \in S$, then there is an $O(M(\|V\|) \cdot \log \|V\|)$ time algorithm that computes a shortest cycle $C$ such that the length of $C$, modulo $m$, is in $S$.

Proof If the precondition of Theorem [3.4 holds, every closed walk whose length, modulo $m$, belongs to $S$, is a cycle or decomposes into cycles such that the length of at least one of these cycles, modulo $m$, belongs to $S$. Hence the problem reduces to finding a shortest closed walk whose length, modulo $m$, belongs to $S$.

Let $G=(V, E)$, where $V=\left\{v_{1}, \ldots, v_{n}\right\}$. For every $r \in\{0, \ldots, m-1\}$ and $0<k \leq n$, we define the boolean matrix $A_{k, r}$ by $A_{k, r}(i, j) \stackrel{d f}{=} 1$ iff there is a walk of length $\ell$ from $v_{i}$ to $v_{j}$ in $G$ with $0<\ell \leq k$ and $\ell \equiv r(\bmod m)$. With $O(\log n)$ boolean matrix multiplications we can determine $k_{\min }$, the length of the desired closed walk. The value of $k_{\min }$ equals the smallest $k$ with $A_{k, r^{\prime}}(i, i)=1$ for some $i \in\{1, \ldots, n\}$ and $r^{\prime} \in S$. First, compute the matrices $A_{k, r}$ where $k$ is a power of 2 , using the identity

$$
A_{2 k, r}=\bigvee_{i=0}^{m-1}\left(A_{k, i} \wedge A_{k, r-i}\right) \vee A_{k, r}
$$

where $\wedge$ and $\vee$ stand for boolean matrix multiplication and componentwise 'or', respectively. Note that $A_{1,1}$ is the adjacency matrix of $G$, and $A_{1, r}, r \neq 1$, is a zero matrix. After that, apply binary search to determine $k_{m i n}$, and a representation of $A_{k_{\text {min }}, r^{\prime}}$ as product of matrices $A_{k, r}$ with $k$ being a power of 2 . A specific closed walk with length $k_{\min }$ (which we know, is a cycle) can now easily be found in additional $O\left(\|V\|^{2}\right)$ time.

\section{Cycle Length Modularity Problems in Undirected Graphs}

In this section, we study the complexity of problems $(S, m)$-UC, for different $S$ and $m$. The case when $S=\{0\}$ has been shown to be in P by Thomassen Tho88. We extend Thomassen's result and prove that for all $S$ such that $0 \in S,(S, m)$-UC is in $\mathrm{P}$.

Theorem 4.1 For each $m$, and each $S \subseteq\{0, \ldots, m-1\}$ such that $0 \in S,(S, m)-\mathrm{UC} \in \mathrm{P}$.

The proof of Theorem 4.1 is an extension of the proof of Thomassen's result for $(\{0\}, m)$-UC, which in turn is based on the result from Robertson and Seymour RS86 for the $k$-disjoint paths problem. We will need the following results related to tree-widths for the proof of Theorem 4.1 Treewidth is an invariant of graphs that has been a central concept in the development of algorithms for fundamental problems in graph theory. See RS86 for a definition of tree-width, and RS85 for a survey on graph minor results. We will not define tree-widths because the definition is rather involved and for the proof of Theorem 4.1 we need know only the following fact about tree-widths of graphs.

Theorem $4.2[R S 86$ For each $t \in \mathbb{N}$, there is a polynomial-time algorithm for deciding whether an undirected graph has tree-width at least $t$.

The following theorem shows that, for fixed $m$, all graphs of sufficiently large tree-width have a cycle whose length is a multiple of $m$.

Theorem 4.3 Tho88] For each $m$, there exists a $t_{m} \in \mathbb{N}$ such that, for each undirected graph $G$ with tree-width at least $t_{m}, G$ contains a cycle of length $\equiv 0(\bmod m)$. 
Roughly speaking, Theorem 4.3 allows us to handle those graphs that have large tree-widths. Theorem 4.5 allows us to handle small tree-widths.

Definition 4.4 For each $t, m \in \mathbb{N}, d_{1}, d_{2}, \ldots d_{k}$ such that, for each $1 \leq i \leq k$, $d_{i}<m$, DISJ-PATH $\left\langle t, m, d_{1}, d_{2}, \ldots, d_{k}\right\rangle$ is defined as follows: DISJ-PATH $\left\langle t, m, d_{1}, d_{2}, \ldots, d_{k}\right\rangle=$ $\left\{\left\langle G, x_{1}, y_{1}, \ldots, x_{k}, y_{k}\right| G\right.$ is an undirected graph such that (a) $G$ has tree width at most $t$, (b) for each $i, x_{i}$ and $y_{i}$ are vertices in $G$, and $(c)$ there exist $k$ node-disjoint paths $P_{1}, P_{2}, \ldots, P_{k}$ in $G$ such that, for each $1 \leq i \leq k, P_{i}$ is a path connecting $x_{i}$ and $y_{i}$ such that $P_{i}$ has length $\left.d_{i}(\bmod m)\right\}$.

Theorem 4.5 Tho88] Let $t, m, d_{1}, d_{2}, \ldots, d_{k} \in \mathbb{N}$ be such that, for each $1 \leq i \leq k, d_{i}<m$. Then, DISJ-PATH ${ }_{\left\langle t, m, d_{1}, d_{2}, \ldots, d_{k}\right\rangle}$ is in $\mathrm{P}$.

Proof of Theorem 4.1, Let $m$ and $S$ be such that $m \in \mathbb{N}, S \subseteq\{0,1, \ldots, m-1\}$, and $0 \in S$. We will now describe a polynomial-time algorithm that decides $(S, m)$-UC. Let $G$ be the input graph. Check, using algorithm in Theorem 4.2 if $G$ has tree-width at least $t_{m}$, where $t_{m}$ is as in Theorem 4.3 If so, then, by Theorem $4.3 G$ has a cycle of length $0(\bmod m)$. Otherwise, $G$ has tree-width at most $t_{m}$. So, we use Theorem 4.5 to check if $G$ has a cycle of length $\ell$ such that $\ell \in S$. For all distinct vertices $v_{1}, v_{2}, v_{3}, v_{4}$ in $G$ such that $\left\{\left(v_{1}, v_{2}\right),\left(v_{3}, v_{4}\right)\right\} \subseteq E(G)$, for each $\ell \in S$, and for each $0 \leq d_{1}, d_{2} \leq m-1$ such that $d_{1}+d_{2}+2=\ell(\bmod m)$, we do the following. Check, using Theorem 4.5] whether there are 2 disjoint paths $P_{1}$ and $P_{2}, P_{1}$ between $v_{1}$ and $v_{3}$ of length $d_{1}(\bmod m)$ and $P_{2}$ between $v_{2}$ and $v_{4}$ of length $d_{2}(\bmod m)$. If there are such disjoint paths, then there is a cycle of length $d_{1}+d_{2}+2=\ell(\bmod m)$, namely the cycle consisting of the edges in $P_{1}$, the edges in $P_{2}$, and the edges $\left(v_{1}, v_{2}\right)$ and $\left(v_{3}, v_{4}\right)$. Note that we may be missing cycles consisting of 3 nodes or less, but that can be easily handled by checking brute-force for all cycles of 3 nodes or less.

Let us consider the complements of the following cycle length modularity problems (for fixed $m \geq 2$ and fixed $0 \leq r<m):(\{0,1, \ldots, m-1\}-\{r\}, m)$-UC. For any $m$ and $r$ such that $0 \leq r<m$, these problems asks whether all cycles in the given graph are of length $\equiv r(\bmod m)$. For $m=2$ and $r=0$, this problem is the odd cycle problem in undirected graphs, which as noted in the introduction is easily seen to be in $\mathrm{P}$ based on the simple observation that any closed walk of odd length in a graph must contain a simple cycle of odd length. For $m=2$ and $r=1$, this problem is the even cycle problem, which is also in P APY91. Arkin, Papadimitriou, and Yannakakis in fact prove, via using the properties of triconnected components of graphs, that for each $m$, and each $0 \leq r<m$, finding whether all cycles in a graph are of length $\equiv r(\bmod m)$ can be done in polynomial time.

Theorem 4.6 ([APY91]) For each $m \in \mathbb{N}$, and each $r$ such that $0 \leq r<m,(\{0,1, \ldots, m-1\}-$ $\{r\}, m)-\mathrm{UC} \in \mathrm{P}$.

Corollary $4.7(\{1,2, \ldots, m-1\}, m)-\mathrm{UC} \in \mathrm{P}$.

The following theorem is an analog of Theorem 3.1(ii) for undirected graphs.

Theorem 4.8 For all $m>2$ and $S \subseteq\{1, \ldots, m-1\}$, the following is true: If for all $p \in S$, and all $d_{1}, d_{2}$, such that $0 \leq d_{1}, d_{2}<m$ and $d_{1}+d_{2} \equiv p(\bmod m)$, it holds that $d_{1} \in S$ or $d_{2} \in S$, then $(S, m)-\mathrm{UC} \in \mathrm{P}$.

The proof given for the corresponding statement regarding directed graphs does not work here. The reason is that closed walks in undirected graphs need not decompose properly into cycles. To see why this is true, consider a closed walk $C$ of length 5 in an undirected graph: $v_{1} v_{2} v_{3} v_{4} v_{2} v_{1}$. Note 
that even though $C$ is a closed walk of length 5 , it is neither a cycle nor does it decompose properly into cycles, basically because $v_{1} v_{2} v_{1}$ is not a valid cycle.

In order to prove Theorem 4.8 we reduce the problem to the problem of determining the period of an undirected graphs, which is solvable in polynomial time by the algorithm from Arkin, Papadimitriou, and Yannakakis APY91. We need the following lemma.

Lemma 4.9 For all $m \geq 1$ and $S=\left\{a_{1}, \ldots, a_{n}\right\} \subseteq\{0, \ldots, m-1\}, S \neq \emptyset$, the following is true: If for all $d_{1} \in S, d_{2} \in S$ it holds that $\left(d_{1}+d_{2}\right) \bmod m \in S$, then $S=\{\ell \mid 0 \leq \ell<m$ and $g \mid \ell\}$ for some $g$ with $g \mid m$.

Proof Let $S-\{0\}=\left\{a_{1}, a_{2}, \ldots, a_{n}\right\}$ Let $g=\operatorname{gcd}\left(a_{1}, \ldots, a_{n}, m\right)$. From number theory (see Apo76) we know that there exist $k_{1}, \ldots, k_{n}, k_{n+1} \in \mathbb{Z}$, such that

$$
k_{1} a_{1}+k_{2} a_{2}+\cdots+k_{n} a_{n}+k_{n+1} m=g .
$$

For all $i, 1 \leq i \leq n+1$, let

$$
k_{i}^{\prime} \stackrel{d f}{=} k_{i}+m\left|k_{i}\right|
$$

Then

$$
\left(k_{1}^{\prime} a_{1}+\cdots+k_{n}^{\prime} a_{n}\right) \bmod m=g,
$$

where $k_{1}^{\prime}, \ldots, k_{n}^{\prime} \geq 0$.

For all $d_{1} \in S, d_{2} \in S$ it holds that $\left(d_{1}+d_{2}\right) \bmod m \in S$. Hence Eq. (4.a) implies that $g \in S$. Furthermore, $s g \in S$ for all $s \in \mathbb{Z}$ such that $0 \leq s g<m$.

Since for each $1 \leq i \leq n, g \mid a_{i}$, it follows that

$$
S=\{\ell \mid 0 \leq \ell<m \text { and } g \mid \ell\} .
$$

This concludes the proof of Lemma 4.9

Proof of Theorem 4.8, Let $\bar{S} \stackrel{d f}{=}\{0, \ldots, m-1\}-S$. Lemma 4.9] implies that

$$
\bar{S}=\{\ell \mid 0 \leq \ell<m \text { and } g \mid \ell\}
$$

for some $g$ with $g \mid m$. Hence

$$
S=\{\ell \mid 0 \leq \ell<m \text { and } g \nmid \ell\} .
$$

Define

$$
S^{\prime}=\{1, \ldots, g-1\}
$$

Since $g \mid m$ holds

$$
x \bmod m \in S \Longleftrightarrow x \bmod g \in S^{\prime}
$$

for all $x \in \mathbb{N}$. Hence $(S, m)$-UC is equivalent to $(\{1, \ldots, g-1\}, g)$-UC. However, $(\{1, \ldots, g-1\}, g)$-UC is the set of graphs containing a cycle not divisible by $g$, which is in $\mathrm{P}$ since the period of a graph (the gcd of all cycle lengths) can be determined in polynomial time APY91. This concludes the proof of Theorem 4.8 . 


\section{Conclusion and Open Problems}

In this paper, we studied the complexity of cycle length modularity problems. We completely characterized (i.e., as either polynomial-time computable or as NP-complete) each problem $(S, m)$-DC, where $0 \notin S$. We also proved that, for each $S$ such that $0 \in S,(S, m)$-UC is in P, and we proved a sufficient condition on $S$ and $m$ for the problem $(S, m)$-UC $(0 \notin S)$ to be in $\mathrm{P}$. We mention several open problems.

1. Theorem 3.1 completely characterizes all modularity problems in directed graphs when $0 \notin S$. Robertson, Seymour, and Thomas RST99 prove that $(\{0\}, 2)-\mathrm{DC}$ is in P. In light of these results, it is natural to ask if $(\{0\}, m)-\mathrm{DC} \in \mathrm{P}$, for some or all $m>2$. Also, the complexity of $(S, m)$-DC such that $0 \in S$ and $m>2$ is still open, except for trivial $(S=\{0,1, \ldots, m-1\})$ cases.

2. Theorem 4.1 shows that all cycle length modularity problems in undirected graphs $(S, m)$-UC such that $0 \in S$ are solvable in polynomial time. What about the complexity of the $(S, m)$-UC problems with $0 \notin S$ which are not covered by Theorem 4.6 or 4.8.

3. Theorem 4.6] shows that, for undirected graphs, the problem of finding whether all cycles have length $\equiv r(\bmod m)$ is in $\mathrm{P}$. What is the complexity of the corresponding problem for directed graphs?

\section{References}

[Apo76] T. Apostol. Introduction to Analytic Number Theory. Undergraduate Texts in Mathematics. Springer-Verlag, 1976.

[APY91] E. Arkin, C. Papadimitriou, and M. Yannakakis. Modularity of cycles and paths in graphs. Journal of the ACM, 38(2):255-274, April 1991.

[BV73] Y. Balcer and A. Veinott. Computing a graph's period quadratically by node condensation. Discrete Mathematics, 4:295-303, 1973.

[EP65] P. Erdös and L. Pósa. On independent circuits contained in a graph. Canadian Journal on Mathematics, 17:347-352, 1965.

[FHW80] S. Fortune, J. Hopcroft, and J. Wyllie. The directed subgraph homeomorphism problem. Theoretical Computer Science, 10:111-121, 1980.

[GL96] A. Galluccio and M. Loebl. Cycles of prescribed modularity in planar digraphs. Journal of Algorithms, 21:51-70, 1996.

[Kas67] P. Kasteleyn. Graph theory and crystal physics. In F. Harary, editor, Graph Theory and Theoretical Physics, pages 43-110. Academic Press, New York, 1967.

[Knu73] D. Knuth. Strong components. Technical Report 004639, Computer Science Department, Stanford University, Stanford, California, 1973.

[MRST97] W. McCuaig, N. Robertson, P. Seymour, and R. Thomas. Permanents, pfaffian orientations, and even directed circuits. In Proceedings of the 29th ACM Symposium on Theory of Computing, pages 402-405, 1997.

[Pol13] G. Polya. Aufgabe 424. Arch. Math. Phys., 20(3):271, 1913.

[RS85] N. Robertson and P. Seymour. Graph minors - a survey. In I. Anderson, editor, Surveys in Combinatorics 1985: Invited Papers for the Tenth British Combinatorial Conference, pages 153-171. Cambridge University Press, 1985. 
[RS86] N. Robertson and P. Seymour. Graph minors II. Algorithmic aspects of tree-width. Journal of Algorithms, 7:309-322, 1986.

[RST99] N. Robertson, P. Seymour, and R. Thomas. Permanents, pfaffian orientations, and even directed circuits. Annals of Mathematics, 150:929-975, 1999.

[Tar72] R. Tarjan. Depth first search and linear graph algorithms. SIAM Journal on Computing, 2:146-160, 1972.

[Tho88] C. Thomassen. On the presence of disjoint subgraphs of a specified type. Journal of Graph Theory, 12(1):101-111, 1988.

[VY89] V. Vazirani and M. Yannakakis. Pfaffian orientations, 0-1 permanents, and even cycles in directed graphs. Discrete Applied Mathematics, 25:179-190, 1989.

[YZ97] R. Yuster and U. Zwick. Finding even cycles even faster. SIAM Journal on Discrete Mathematics, 10(2):209-222, May 1997. 